\title{
Polar Solvation and Electron Transfer DE-FG02-89ER14020
}

\author{
Annual Progress Report for \\ Budget Period 7/1/92-6/30/93 \\ Prepared 4/12/93
}

DOE/ER/14020--4

DE93 013328

1) Completion of Previous Studies on Solvation Dynamics: In past reports I have mentioned our studies of the probe dependence of solvation dynamics. We have been collecting time-resolved fluorescence data on a wide variety of solvation probes in a single reference solvent (1-propanol at $253 \mathrm{~K}$ ) in order to understand what role the solute plays in determining the time-dependence of solvation. We have now completed the experimental part of this exercise, having made measurements on 16 different probes. While the solvation times of most solutes considered span a relatively narrow range, we find that simple aromatic amines fall into a distinct class with much faster (2-3 times) dynamics. We are still in the process of trying to understand this difference through the use of computer simulations. It currently appears that the difference is due to the relative localization of the charge change in the simple aromatic amines relative to other solutes with more complicated polar groups. We intend to begin writing up this comparison very shortly.

Related experimental work concerns how to assess the correct time-zero spectrum in solvation dynamics measurements. In order to compare experimental measurements of the solvation response to theory or simulation it is important to assess how limits on instrumental time resolution influences the results. On the basis of our "Inhomogeneous Kinetics" work [J. Phys. Chem. 95, 5170 (1991)] we have developed a method that allows one to calculate where the fluorescence spectrum should be immediately after excitation. We have made extensive tests of the method and are now confident that the correct $t=0$ spectrum can be estimated to within $\pm 200 \mathrm{~cm}^{-1}$ (typically this means to within $\pm 10 \%$ of the total shift.) This ability is crucial to our being able to understand the very fast inertial components of solvation that we discuss in Part 3 below. As part of these experiments we have made many measurements of the excitation-wavelength dependence of the solvation response. We find that while the amplitude of the solvation response varies (as predicted by our model) with excitation wavelength, the time dependence of the response does not. This answers a long standing question we have had concerning the linearity or nonlinearity of the solvation response. At least to the extent that we can use the excitation wavelength to vary the subset of solvation states that we initially excite, we find the dynamics to be independent of this selection and to therefore be linear (or homogeneous). This behavior is in marked contrast to the behavior we observed in ionic solutions [J. Phys. Chem. 95, 9095 (1991)]. We are also (finally) in the process of finishing a manuscript on this time-zero work.

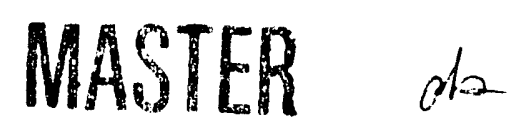

OISTRIBUTION OF THIS DOCLIMENT IS UNIIMITER 
2) Dipole Lattice Studies: In the last year we have completed two further papers (enclosed) involving simulations of solvation in dipole lattice solvents [first discussed in J. Chem. Phys. 95, 9219]. The first paper, Solvation Dynamics in a Brownian Dipole Lattice: A Comparison between Theory and Computer Simulation, uses the simulation results, along with results of dielectric dispersion calculations of Zhou and Bagchi to make a detailed comparison between dynamics observed in this simple model solvent and the dynamics predicted by analytical theories of solvation currently available. In the rotation only / purely diffusive regime represented by these lattice solvents, we observed that most theories, even simple continuum models, do a reasonable job of predicting the average time scale of the solvation response. However, only models that explicitly consider the molecular nature of the solvent are able to describe the non-exponential time-dependence of the response. We find that two molecular models, the dynamical MSA model of Wolynes [J. Chem. Phys., 86, 5133 (1987)] and the memory function theory of Fried and Mukamel [J. Chem. Phys. 93, 932 (1990)], provide essentially exact predictions of the simulated dynamics (when properly applied.)

In the second paper, On the Validity of the "Inverted Snowball" Picture of Solvation Dynamics, we use further dipole lattice simulations to explore the spatial dependence of the solvation response. The folklore in this field is that different local regions of the solvent respond at different rates to a solute perturbation. Those regions closest to the solvent are thought to respond most slowly via what has become known as the Onsager "inverted snowball" effect. Our simulations clearly show that this popular picture of the solvation response is incorrect. These results point out the futility of any attempt to decompose the solvation response into independent contributions from different solvents regions. Hopefully, this paper will help to clear up a well-entrenched misconception about how one should picture the solvation response.

3) Inertial Components of the Solvation Response: Simulation studies show that for small solvents such as water and acetonitrile solvation dynamics may be dominated by very fast inertial components. It has only been very recently that such fast inertial components have (apparently) been observed in experiment. Since the presence of such motions is crucial to determining how polar solvents couple to charge-transfer reactions we are very interested in characterizing the extent and the time scale of such motions in a variety of solvents. We have been taking several approaches to this end. First we have been working closely in collaboration with Graham Fleming at the University of Chicago since his group was the first to observe these components in experiment. We have used MD simulations to directly model those systems that the Chicago group has been able to study experimentally. We are presently working on a manuscript comparing the experimental and simulated dynamics of several solutes in acetonitrile and methanol. The results of this first study have been encouraging. Although there is considerable uncertainty associated with both experiment and simulation, the agreement between the two approaches is quite good. Both the time dependence of the slower dynamics as well as the relative amplitude of fast and slow dynamics appears to be accurately modeled in simulation. 
In order to push this work further we have constructed a fluorescence upconversion spectrometer of design similar to the one used by Fleming and coworkers. We are currently in the process of reconstructing the system after moving it into another laboratory. When finished (a few more days now) we expect to achieve a time resolution of $\sim 100 \mathrm{fs}$-- the best currently obtainable with such an instrument. Such resolution will be sufficient to observe the inertial components of many simple liquids. In the future (possibly as soon as this summer) we plan to upgrade the laser so as to hopefully get down to an ultimate resolution of $-50 \mathrm{fs}$. This kind of time resolution, in conjunction with our method for estimating the true position of the time-zero spectrum, should enable us to completely characterize the fast parts of the solvation response that have largely been missed in previous studies. We will simultaneously be performing molecular dynamics simulations of these systems in order to help better interpret the observed dynamics.

4) Simple Models of Solvation Dynamics: One of our most significant accomplishments over the last year has been the development of a simplified model for understanding solvation dynamics in terms of the reorientational dynamics of solvent molecules. The paper entitled A Simple Interpretation of Polar Solvation Dynamics provides a description of the initial model. Based on a consideration of the fast inertial solvation response discussed above, we observed that the (collective) solvation response could be expressed as a simple function of the single-particle reorientational time-correlation function (tcf) of solvent molecules in the absence of the solute. The connection between the two depends on the polarity (dipole density) of the solvent in such a way that for a given single-particle dynamics, the higher the solvent polarity the faster the solvation response. This relation between the speed of solvation and the solvent polarity is present in all reasonable theories of solvation dynamics. However, the physical meaning of this connection is laid bare in a particularly simple and intuitive manner in the present model. We find that the solvent polarity serves as a direct translation factor between the extent of molecular motion and its effect on the solvation energy. This polarity dependence comes about due to the fact that the more polar the solvent the more solvent-solvent interactions serve to suppress the solvent's orientational response to changes in the solute. The greater this suppression the less that solvent molecules have to move to change the solvation energy and thus the faster the response.

In our initial study we considered only solvation of simple ionic solutes and assumed that only reorientational dynamics of solvent molecules contributes to the response. The ability to predict the simulated solvation dynamics from a knowledge of the rotational tcfs alone was remarkably good. Good enough in fact that we can use this model to make predictions for what sorts of inertial dynamics to expect in experimental solvents. Such predictions will serve as a guide for the experimental work described above. We also intend to push the theory further and see if we can make accurate predictions about more complex solutes as well as understand why translational motions appear to be unimportant in the response. This theoretical approach should serve as an important adjunct to the theories of dynamics currently being developed by several groups using an instantaneous normal mode perspective. 
5) Rotational Dynamics and Dielectric Friction: We have also been engaged in two studies related to how polar interactions affect rotational dynamics of solvent molecules, a phenomenon known as dielectric friction. In the first project, which is now nearing completion, we have used Brownian dipole lattice simulations to test the accuracy of current theories of dielectric friction and their connection with solvation dynamics. By changing the time scales of the solute and solvent motion we have observed a variety of interesting regimes only some of which conform to the predictions of available theory. We are in the process of trying to understand why available theories do not work well in some regimes and to develop more predictive models for the dynamics in these cases.

In a related study we have measured the rotational dynamics of the solvatochromic probe coumarin 102 in the presence of strongly hydrogen bonding alcohols. In the paper Rotational Diffusion of Coumarin 102 in Trifluoroethanol: The Case for Solvent Attachment we show that in some cases what has previously been interpreted as a nonspecific dielectric friction effect may be better viewed in terms of long-lived solute-solvent attachment.

6) Intramolecular Electron Transfer Reactions: We have recently begun work on two projects involving intermolecular electron transfer in solution. The first involves studies designed to test the predicted relationship between the rate of electron transfer and the solvation rate. Although a number of theories predict that the two phenomena should be closely linked, few convincing demonstrations of this connection have been reported. In collaboration with Professor Maslak of Penn State we are trying to design intramolecular donor acceptor systems in which the influence of solvent dynamics on the rate of intramolecular charge transfer will be clear. Thus far we have examined several possible systems with somewhat disappointing results. For the compounds synthesized to date intramolecular flexibility has made interpretation of the fluorescence decay kinetics in terms of electron transfer rates difficult. To proceed further we are considering more complicated syntheses that will provide less flexible compounds where the decay kinetics should be much simpler.

The second area of study involves time-resolved fluorescence measurements of the twisted intramolecular charge transfer (TICT) process. Although molecules undergoing this process have been studied for many years the how the intramolecular twisting that accompanies such reactions is coupled to the solvent surroundings is poorly understood. We have observed that reactions of some TICT molecules exhibit inhomogeneous kinetics at low temperatures and we believe that studies of such cases along with fluorescence depolarization measurements will help to better elucidate the nature of this dynamical coupling. We have just finished synthesizing several compounds that we will use in this work and preliminary measurements in supercritical fluids look quite interesting. 
Z) Intermolecular Donor-Acceptor Complexes: The final project we have been involved in during the past year entails studies of intermolecular electron transfer in electron donor acceptor (EDA) complexes. We have begun to explore the behavior of a number of such complexes that emit measurable fluorescence at room temperature. Our original intent was to study electron transfer in the strongly inverted region by viewing the non-radiative decay rates of these $s_{p}$ iecies which reflects the rate of back electron transfer. Guilford Jones II ar. I coworkers had observed solvent-dependent isotope effects on the fluorescence of some EDA complexes and we thought that time-resolved studies of these systems would help to better understand the involvement of vibrational modes in electron transfer in the inverted regime. To our surprise we found that non-exponential fluorescence decays are a common occurrence in EDA complexes. We have already performed a large number of experiments to understand why. It appears that after chargetransfer excitation some type of higher-order association (i.e. formation $\mathrm{DA}_{2}$ or $\mathrm{D}_{2} \mathrm{~A}$ species) occurs even under relatively dilute conditions. The association appears to be faster than would be expected on the basis of simple diffusion alone and we are currently trying to understand this puzzling observation. An understanding of this phenomenon is obviously important if one is to use EDA complexes to study the electron transfer process itself.

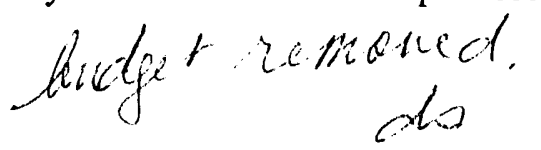

\section{DISCLAIMER}

\footnotetext{
This report was prepared as an account of work sponsored by an agency of the United States Government. Neither the United States Gnvernment nor any agency thereof, nor any of their employees, makes any warranty, express or implied, or assumes any legal liability or responsibility for the accuracy, completeness, or usefulness of any information, apparatus, product, or process disclosed, or represents that its use would not infringe privately owned rights. Reference herein to any specific commercial product, process, or service by trade name, trademark, manufacturer, or otherwise does not necessarily constitute or imply its endorsement, recommendation, or favoring by the United States Government or any agency thereof. The views and opinions of authors expressed herein do not necessarily state or reflect those of the United States Government or any agency thereof.
} 

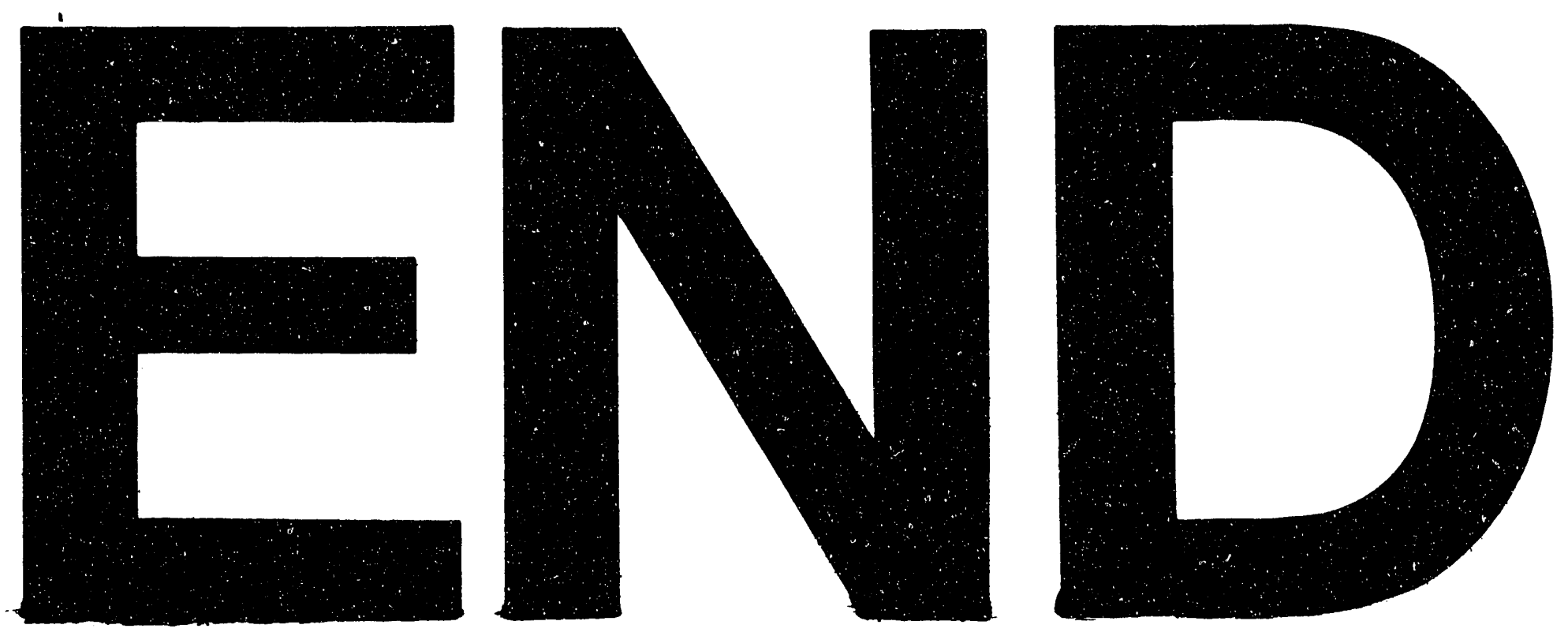

Ex

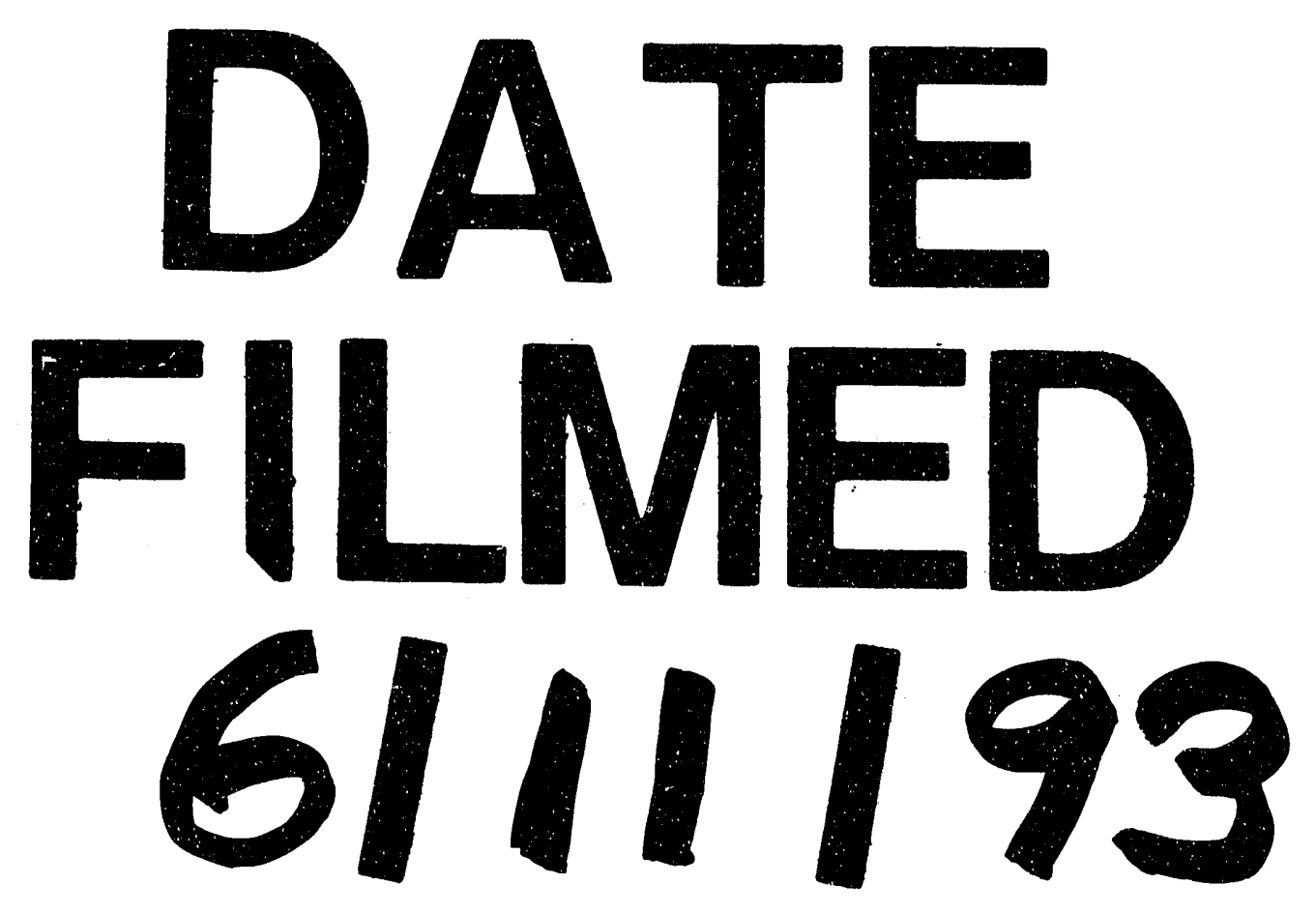


